J. Dairy Sci. 98:5018-5018

http://dx.doi.org/10.3168/jds.2015-98-7-5018

(C) American Dairy Science Association ${ }^{\circledR}, 2015$.

\title{
Erratum to "Effect of technical cashew nut shell liquid on rumen methane emission and lactation performance of dairy cows" (J. Dairy Sci. 98:4030-4040)
}

\section{A. F. Branco, F. Giallongo, T. Frederick, H. Weeks, J. Oh, and A. N. Hristov}

In Table 1 (page 4032), the value for organic matter (OM) was incorrect. The corrected table is shown below (corrected value in bold).

The journal regrets the error.

\section{REFERENCES}

Branco, A. F., F. Giallongo, T. Frederick, H. Weeks, J. Oh, and A. N. Hristov. 2015. Effect of technical cashew nut shell liquid on rumen methane emission and lactation performance of dairy cows. J. Dairy Sci. 98(6):4030-4040.

Table 1. Ingredient and chemical composition (\% of DM unless noted otherwise) of the basal diet fed to dairy cows

\begin{tabular}{|c|c|}
\hline Item & $\%$ of DM \\
\hline \multicolumn{2}{|l|}{ Ingredient composition } \\
\hline Corn silage $^{1}$ & 43.5 \\
\hline Alfalfa haylage $^{2}$ & 15.0 \\
\hline Grass hay & 4.4 \\
\hline Cottonseed, hulls & 2.2 \\
\hline Corn grain, ground & 9.0 \\
\hline Candy by-product meal ${ }^{3}$ & 5.9 \\
\hline Soybean seeds, whole, heated & 6.2 \\
\hline Canola meal, mechanically extracted & 6.1 \\
\hline Molasses & 4.2 \\
\hline Optigen $^{4}$ & 0.5 \\
\hline Mineral-vitamin premix $^{5}$ & 3.0 \\
\hline \multicolumn{2}{|c|}{ Analyzed, except $\mathrm{NE}_{\mathrm{L}}$, chemical composition ${ }^{6}$} \\
\hline $\mathrm{DM}$ & 52.2 \\
\hline OM & 92.9 \\
\hline $\mathrm{CP}$ & 15.2 \\
\hline $\mathrm{NDF}$ & 31.3 \\
\hline $\mathrm{ADF}$ & 21.4 \\
\hline Starch $^{7}$ & 29.8 \\
\hline $\mathrm{Ca}$ & 0.80 \\
\hline $\mathrm{P}$ & 0.34 \\
\hline $\mathrm{NE}_{\mathrm{L}}{ }^{8} \mathrm{Mcal} / \mathrm{kg}$ & 1.53 \\
\hline
\end{tabular}

${ }^{1}$ Corn silage contained (DM basis) $7.0 \% \mathrm{CP}, 41.5 \%$ starch, $33.5 \%$ $\mathrm{NDF}, 21.4 \% \mathrm{ADF}, 0.17 \% \mathrm{Ca}$, and $0.22 \% \mathrm{P}$.

${ }^{2}$ Alfalfa haylage contained (DM basis) $18.8 \% \mathrm{CP}, 43.4 \% \mathrm{NDF}, 37.8 \%$ $\mathrm{ADF}, 1.16 \% \mathrm{Ca}$, and $0.32 \% \mathrm{P}$.

${ }^{3}$ Candy by-product meal (Graybill Processing, Elizabethtown, PA) contained (DM basis) $17 \% \mathrm{CP}$ and $27.8 \% \mathrm{NDF}$.

${ }^{4}$ Nonprotein nitrogen source $(243.2 \%$ CP, DM basis) from Alltech Inc. (Nicholasville, KY).

${ }^{5}$ The premix (Cargill Animal Nutrition, Cargill Inc., Roaring Spring, $\mathrm{PA})$ contained (\%, as-is basis) trace mineral mix, 0.86; $\mathrm{MgO}, 8.0$; $\mathrm{NaCl}, 6.4$; vitamin ADE premix (Cargill Animal Nutrition), 0.48; limestone, 37.2; selenium premix (Cargill Animal Nutrition), 0.07; and dry corn distillers grains with solubles, 46.99; Ca, $14.1 \%$;, $0.39 \%$; $\mathrm{Mg}, 4.59 \%$; K, 0.44\%; S, 0.39\%; Se, $6.91 \mathrm{mg} / \mathrm{kg}$; Cu, $362 \mathrm{mg} / \mathrm{kg} ; \mathrm{Zn}$, $1,085 \mathrm{mg} / \mathrm{kg}$; Fe, $186 \mathrm{mg} / \mathrm{kg}$, vitamin A, 276,717 IU $/ \mathrm{kg}$; vitamin D, $75,000 \mathrm{IU} / \mathrm{kg}$; and vitamin E, 1,983 IU $/ \mathrm{kg}$.

${ }^{6}$ Analyzed by Cumberland Valley Analytical Services (Maugansville, MD) using wet chemistry methods.

${ }^{7}$ Analyzed according to Bach Knudsen (1997).

${ }^{8}$ Estimated based on NRC (2001). 